\title{
EXOSTOSIS AUDITIVA EXTERNA EN INDIVIDUOS ADULTOS DEL HOLOCENO TARDÍO (1500 AP - SIGLO XIX) EN PATAGONIA AUSTRAL
}

\author{
EXTERNAL AUDITORY EXOSTOSIS IN ADULT INDIVIDUALS FROM LATE \\ HOLOCENE (1500 AP - 19TH CENTURY) IN SOUTHERN PATAGONIA
}

\author{
Cynthia Daniela Pandiani *, Jorge Alejandro Suby ${ }^{1,2}$ y Ana Luisa Santos ${ }^{3}$ \\ ${ }^{1}$ Grupo de Investigación en Bioarqueología. Unidad de Enseñanza Universitaria Quequén. Departamento de Arqueología. Univer- \\ sidad Nacional del Centro de la Provincia de Buenos Aires. Quequén. Argentina \\ ${ }^{2}$ INCUAPA-CONICET \\ ${ }^{3}$ Department of Life Sciences. Centro de Investigação em Antropologia e Saúde (CIAS). Universidad de Coimbra. Coimbra. Portugal
}

PALABRAS CLAVE torus auditivus; cazadores-recolectores; Argentina; Chile

RESUMEN La exostosis auditiva externa (EAE) es una elevación ósea del conducto auditivo externo. Se asocia con la exposición a aguas frías, aunque también con bajas temperaturas, viento, traumas e infecciones repetitivas. Su prevalencia fue poco estudiada en poblaciones del pasado en Patagonia Austral. El objetivo de este trabajo fue estimar la frecuencia de EAE de acuerdo con la región, sexo, edad de muerte y tipo de economía en una muestra de 24 cráneos de individuos adultos (18 masculinos, 5 femeninos y uno indeterminado) pertenecientes al Holoceno Tardío. Se determinó la presencia, tamaño, localización, forma y lateralidad de la EAE. El 79,2\% (n=19) de los individuos analizados presentó EAE, bilateral en el 56, 3\% (9/16), en general, de tamaño pequeño y mediano $(73,7 \%$ y $21,1 \%$, respectivamente). La EAE resultó más frecuente en individuos masculinos y adultos medios, sin presentar diferencias significativas. La prevalencia de EAE fue mayor en esqueletos asociados a dietas terrestres (58,3\%), en especial, en los de Santa Cruz/Magallanes (33,3\%) y norte de Tierra del Fuego (25\%), aunque sin diferencias significativas, mientras que en la costa y el interior son similares. Los resultados preliminares sugieren una combinación de factores ambientales (agua fría, viento y temperatura) como causa de la EAE en Patagonia Austral, aunque son necesarias evaluaciones sobre muestras más amplias y acotadas temporalmente. Rev Arg Antrop Biol 21(1), 2019. doi:10.17139/raab.2019.0021.01.05

\section{KEY WORDS torus auditivus; hunter-gatherers; Argentina; Chile}

ABSTRACT External auditory exostosis (EAE) is a new bone formation of the external auditory canal. It is particularly associated with cold water exposition, but also with low environmental temperatures, wind, trauma, and repetitive infections. Its prevalence has been scarcely studied in past human populations from Southern Patagonia. The aim of this work was to study the prevalence of EAE and its possible relationship to region, sex, age-at-death, and economic strategies in a sample of 24 skulls of Late Holocene adult individuals (18 males, 5 females, and 1 undetermined) from Southern Patagonia. Presence, size, location, form, and laterality of the EAE were determined. In the studied sample, $79.2 \%(n=19)$ of individuals were affected by EAE, bilateral in $56.3 \%(9 / 16)$, of small and middle size $(73.7 \%$ and $21.1 \%$, respectively).

La exostosis auditiva externa (EAE), también denominada torus auditivus, es una elevación ósea de la entrada o el tracto medio del conducto auditivo externo (Arnay de la Rosa, Velasco-Vazquez, González Reimers y Santolaria-Fernandez, 2001; Deleyiannis, Cockcroft y Pinczower, 1996; House y Wilkinson, 2008; Pezo Lanfranco L., Pezo Lanfranco S. y Eggers, 2009; Velasco-Vazquez, Betancor-Rodriguez, Arnay de la Rosa y Gonzalez-Reimers, 2000). Macroscópicamente está caracterizada por la formación de hueso
EAE was more frequent in males and middle adults, without presenting significant differences. Similar prevalence of EAE was observed in individuals from coastal and inland regions. However, this was more pronounced in individuals with a terrestrial diet $(58.3 \%)$, in particular, those from Santa Cruz/Magellan region (33.3\%) and from the north of Tierra del Fuego (25\%); no significant differences were found with respect to those from southern Tierra del Fuego. These first results suggest the combination of environmental factors (cold water, wind, and low environmental temperature) as possible causes for the high frequency of EAE in these individuals from Southern Patagonia. However, further research including larger samples is necessary. Rev Arg Antrop Biol 21(1), 2019. doi:10.17139/raab.2019.0021.01.05

\footnotetext{
* Correspondencia a: Cynthia Daniela Pandiani. Grupo de Investigación en Bioarqueología. Unidad de Enseñanza Universitaria Quequén. Departamento de Arqueología. Universidad Nacional del Centro de la Provincia de Buenos Aires. Calle $508 N^{\circ} 881.7631$ Quequén. Argentina. E-mail: cynthiapandiani@yahoo.com.ar
}

Financiamiento: Proyecto PIP-CONICET 2015$11220150100016 \mathrm{CO}$ otorgado a los Dres. Paula Novellino y Jorge Suby; y PICT 2016-0191, otorgado a los Dres. Jorge Suby y Leandro Luna.

Recibido 18 Diciembre 2017; aceptado 5 Mayo 2018

doi:10.17139/raab.2019.0021.01.05 
en forma esferoide $u$ oval, mientras que a nivel microscópico se define por la formación de estructuras compactas de hueso lamelar (Fowler y Osmun, 1942; Graham, 1979; Hutchinson, Denise, Daniel y Kalmus, 1997). Su desarrollo produce elevaciones del hueso temporal, que involucran el hueso timpánico, hasta obstruir el canal auditivo por completo en los casos más severos (DiBartolomeo, 1979; González Reimers, Lorenzo de la Peña, Sarmiento-Herrera, Pérez Piñero y Arnay de la Rosa, 2008). Debido a esos cambios, puede ocasionar otitis externa repetitiva, cambios de $\mathrm{pH}$ en el epitelio superficial, dermatitis, bloqueo por cerumen y, en grados avanzados, pérdida de la audición (House y Wilkinson, 2008; Hutchinson et al., 1997; Timofeev, Notkina y Smith, 2004).

Inicialmente la EAE fue atribuida a infecciones repetitivas y traumas del conducto auditivo (DiBartolomeo, 1979; Fowler \& Osmun, 1942). Berry y Berry (1967) sugirieron que podría ser hereditaria, por lo que en algunas ocasiones fue empleada como medida de las distancias genéticas entre poblaciones (Hanihara, Ishida y Dodo, 2003). Posteriormente se propuso la hipótesis hidrotérmica (Kennedy, 1986), según la cual la EAE está posiblemente asociada a la inmersión en aguas frías, lo que desencadenaría procesos irritativos e inflamatorios, produciendo la estimulación de la función osteoblástica en el conducto auditivo. Según esta propuesta, la EAE afectaría en mayor frecuencia a individuos que se encuentran entre $30^{\circ}$ y $45^{\circ}$ de latitud $\mathrm{N}$ o $\mathrm{S}$ $\mathrm{y}$ que frecuentarían la explotación de recursos marinos. Por el contrario, tendría un menor desarrollo en poblaciones que habitan entre las latitudes $0^{\circ}$ y $30^{\circ}(\mathrm{N}$ y S $)$ donde el agua es cálida, $\mathrm{y}$ en latitudes mayores a los $45^{\circ}(\mathrm{N}$ y S) donde las inmersiones en agua serían menos frecuentes debido al riesgo de sufrir hipotermia (Kennedy, 1986). Esta hipótesis está actualmente apoyada por estudios clínicos, en particular sobre personas que practican actividades acuáticas como el surf (Deleyiannis et al., 1996; Kroon, Lawson, Derkay, Hoffmann y McCook, 2002) y el buceo (Azizi, 2011), los cuales sugieren que la formación ósea sería proporcional a la frecuencia de exposición en aguas frías de entre $15^{\circ} \mathrm{y}$ $19^{\circ} \mathrm{C}$. Por ejemplo, un examen realizado en 307 surfistas en California reveló una frecuencia de $70 \%$ de EAE (Wong et al., 1999), mientras que
Kroon et al. (2002) indicaron que la prevalencia de EAE es mayor en individuos que practican este deporte en aguas frías $(62 \%)$, sin diferencias significativas entre sexos. Además de la temperatura, la salinidad del agua podría influir en el desarrollo de EAE (Pezo Lanfranco et al., 2009). Sin embargo, sus efectos son discutidos, dado que Harrison (1951) encontró un 5,6\% de prevalencia en nadadores de agua salada, similar a un $5 \%$ en nadadores de agua dulce.

A medida que aumenta la cantidad de años de prácticas acuáticas se incrementaría el grado de obstrucción del canal auditivo externo (Deleyiannis et al., 1996). En este sentido, tanto estudios clínicos (Azizi, 2011; Deleyiannis et al., 1996; Kroon et al., 2002) como bioarqueológicos (Crowe et al., 2010; Mazza, 2016; Standen, Arriaza y Santoro, 1997) muestran una mayor prevalencia de EAE en individuos adultos, en especial en aquellos casos posiblemente vinculados a causas ambientales. Es decir, el tiempo y la frecuencia de estas prácticas serían insuficientes para que se desarrolle la EAE en subadultos (DiBartolomeo, 1979). A su vez, su desarrollo estaría condicionado por el uso de algún medio de protección del canal auditivo (Deleyiannis et al., 1996; Timofeev et al., 2004; Zoltan, Taylor y Achar, 2005).

Los estudios bioarqueológicos realizados hasta el momento sugieren la posible influencia de las bajas temperaturas del agua como explicación de la alta prevalencia de EAE, particularmente en poblaciones cuya economía se basa en la explotación de recursos marinos (Kennedy, 1986; Ponce, Ghidini y GonzálezJosé, 2008; Standen et al., 1997). Sin embargo, trabajos recientes muestran que la EAE también afectó a individuos que habitaron áreas entre 0 y $30^{\circ}$ de latitud Norte y Sur (Castro, Goycoolea \& Silva-Pinto, 2016; Okumura, Boyadjian y Eggers, 2007; Standen et al., 1997; VelascoVazquez et al., 2000; Villotte y Knusel, 2015) y poblaciones no vinculadas a ambientes costeros (Goode, 2009; Okumura et al., 2007; Standen et al., 1997), por lo que otros factores podrían estar involucrados. Particularmente, la acción refrigerante de los vientos fue propuesta como un factor que también propicia la aparición de EAE, debido a la prevalencia diferencial expresada en individuos que desarrollan las mismas actividades acuáticas en aguas de temperatura 
similar (Okumura et al., 2007). En este sentido, Goode (2009) propuso que la acción del viento y la temperatura ambiental, junto con la exposición acuática, habrían influido en la aparición de EAE en una población agrícola-pastoril en Nubia.

A pesar del estudio de la EAE en diversas poblaciones antiguas, son escasos los análisis realizados en poblaciones cazadoras-recolectoras en Argentina. Uno de ellos analizó una muestra de individuos provenientes del bajo $\mathrm{Pa}-$ raná del Holoceno Tardío (Mazza, 2016), cuyos resultados indicaron la presencia de EAE en un $6,25 \%(11 / 176)$ de los esqueletos analizados, todos ellos correspondientes a individuos masculinos adultos. Como único antecedente en $\mathrm{Pa}-$ tagonia Austral y con el objetivo de evaluar la hipótesis hidrotérmica propuesta por Kennedy (1986), Ponce et al. (2008) analizaron la prevalencia de EAE en una muestra de 108 cráneos pertenecientes a individuos que habitaron la Isla Grande de Tierra del Fuego durante el Holoceno Tardío. Entre ellos se registraron seis individuos $(5,5 \%)$ con evidencias de EAE, mientras que el $9,1 \%$ de los individuos vinculados a una economía marina presentaban lesiones compatibles con esta patología. Sin embargo, el tipo de práctica económica de los restos fue inferida a partir de su distribución geográfica basada en datos etnográficos y no se ofrecen detalles de los individuos analizados. Además, tampoco fue detallada la metodología empleada para el diagnóstico de la EAE y sus grados de desarrollo, por lo que nuevas evaluaciones resultan necesarias. Por este motivo, el objetivo de este trabajo es analizar la frecuencia de la EAE en individuos adultos del Holoceno Tardío de Patagonia Austral, explorando su posible relación con el tipo de práctica económica inferida a partir de información paleodietaria.

\section{Características ambientales y etnográficas de Patagonia Austral}

Patagonia Austral es un área extensa y diversa en términos geográficos, ecológicos y climáticos. Se pueden distinguir dos grandes sub-áreas: la andina occidental, caracterizada por un cinturón boscoso, de clima frio, ventoso y un promedio anual de precipitaciones de $500-1000 \mathrm{~mm}$ y la extra-andina oriental, la cual presenta planicies y estepas con precipitaciones anuales de $300 \mathrm{~mm}$ en promedio (Bailey, 1989; McCulloch, Bentley, Tipping \& Clapperton, 2005; Oliva et al., 2001). Ambas sub-regiones poseen un clima frío-templado, con temperaturas medias anuales que varían de $8^{\circ} \mathrm{C}$ en el sector noreste a $4^{\circ} \mathrm{C}$ en el sudoeste (Mancini, 2003; Mancini, Prieto, Paez y Schábitz, 2008). Los vientos predominan del sector oeste y suroeste, presentan variaciones en su intensidad y determinan junto con la cordillera de los Andes el gradiente de precipitaciones (Garreaud, Vuille, Compagnucci y Marengo, 2008). La velocidad media anual del viento es de $31 \mathrm{~km} / \mathrm{h}$, pudiendo haber ráfagas de $50 \mathrm{~km} / \mathrm{h}$ y excepcionalmente de hasta $200 \mathrm{~km} / \mathrm{h}$ (Administración de Parques Nacionales, 2007). Según datos del Servicio de Hidrografía Naval de Argentina, el promedio anual de la temperatura del agua en superficie en la costa es de $8,4^{\circ} \mathrm{C}$, con variaciones entre $13,1^{\circ} \mathrm{C}$ (enero-febrero) y $3,8^{\circ} \mathrm{C}$ (julio-agosto). Por otro lado, el canal de Beagle presenta un promedio anual de $6,3^{\circ} \mathrm{C}$ con variaciones entre $7,5^{\circ} \mathrm{C}$ (enero) y $5,1^{\circ} \mathrm{C}$ (agosto) (Borla y Vereda, 2015).

Los datos etnográficos y etnohistóricos distinguen principalmente cuatro grupos que habitaron Patagonia Austral: los Aonikenk en el continente y Selk'nam en el norte de Tierra del Fuego (cazadores-recolectores terrestres), y los Alakaluf en el litoral suroccidental y Yámanas en el litoral suroriental de Tierra del Fuego (cazadores-recolectores marinos) (Gusinde, 1982, 1986; Saletta, 2014). Abundante evidencia zooarqueológica muestra que el guanaco (Lama guanicoe) jugaba un papel dietario predominante entre los cazadores-recolectores terrestres. Sin embargo, también eran consumidas algunas especies de aves terrestres como el choique (Rhea pennata) y marinas como pingüinos (Spheniscus magellanicus) y cormorán imperial (Phalacrocorax atriceps), además de lobos marinos (Arctocephalus australis y Otaria flavescens) y moluscos (e.g. Mytilus sp., Aulacomya sp.) (Barberena, 2008; Borella, 2010; Cruz, Muñoz y Zangrando, 2007; Cruz, Astete, Nauto y Borrero, 2010; Muñoz, 2011). Además, recolectaban bayas, hongos y algunas hojas (Guisinde, 1982, 1986).

Por el contrario, los cazadores-recolectores marítimos consumían lobos marinos cazados mediante arpones de hueso, y la pesca se destacaba como práctica anual mediante redes o líneas de pesca sin anzuelo verdadero y canoas de corte- 
za, impulsada por remos, que les permitían la exploración del ambiente insular en la búsqueda y captura de recursos (Gusinde, 1982, 1986; Saletta, 2014). Además, recolectaban aves y sus huevos, peces y moluscos, que complementaban con bayas y hongos (Orquera, 2005; Orquera y Piana, 1999; Saletta, 2014; Tessone, Zangrando, Valencio y Panarello, 2003; Tivoli, 2014; Tivoli y Zangrando, 2011; Yesner, Figuerero Torres, Guichón y Borrero, 2003; Zangrando, 2009). Al menos estacionalmente también eran consumidas especies animales terrestres. Las principales fuentes etnográficas no mencionan la recolección de recursos a través del buceo (Gusinde 1982; Orquera y Piana, 1999; Saletta, 2014), aunque esta práctica no puede ser descartada por completo en momentos históricos o previos al contacto.

A pesar de la existencia de economías terrestres y marítimas en escalas macro-regionales, estas evidencias sugieren la existencia de poblaciones con economías intermedias (o mixta), caracterizadas por la ausencia de una predominancia clara de consumo de recursos terrestres o marítimos, en especial en regiones próximas a la costa (Barberena, 2002; Borrero y Barberena, 2006; Yesner et al., 2003).

\section{MATERIAL Y MÉTODOS}

Se evaluó la presencia de EAE en cráneos pertenecientes a individuos adultos del Holoceno Tardío de Patagonia Austral (Tabla 1; Fig. 1). A los fines de este trabajo, consideramos Patagonia Austral como la región que se encuentra al sur del río Santa Cruz (Provincia de Santa Cruz, Argentina). Para ello, se seleccionaron individuos adultos que presentaran los siguientes criterios: al menos uno de los conductos auditivos (CA) en buenas condiciones de conservación; que contaran con información arqueológica sobre su lugar de hallazgo y en lo posible datos sobre su cronología y paleodieta en base a estudios isotópicos del $\mathrm{C}$ y N. Luego de esta selección, la muestra estuvo conformada por 24 cráneos adultos jóvenes y medios (18 masculinos, 5 femeninos y 1 indeterminado), cuyo origen e institución donde se encuentran resguardados se muestra en la Figura 1 y Tabla 1. La edad de muerte y el sexo de los individuos analizados fue la reportada en investigaciones previas (Tabla 1).

La EAE fue diagnosticada cuando el conducto auditivo presentó cualquier modificación

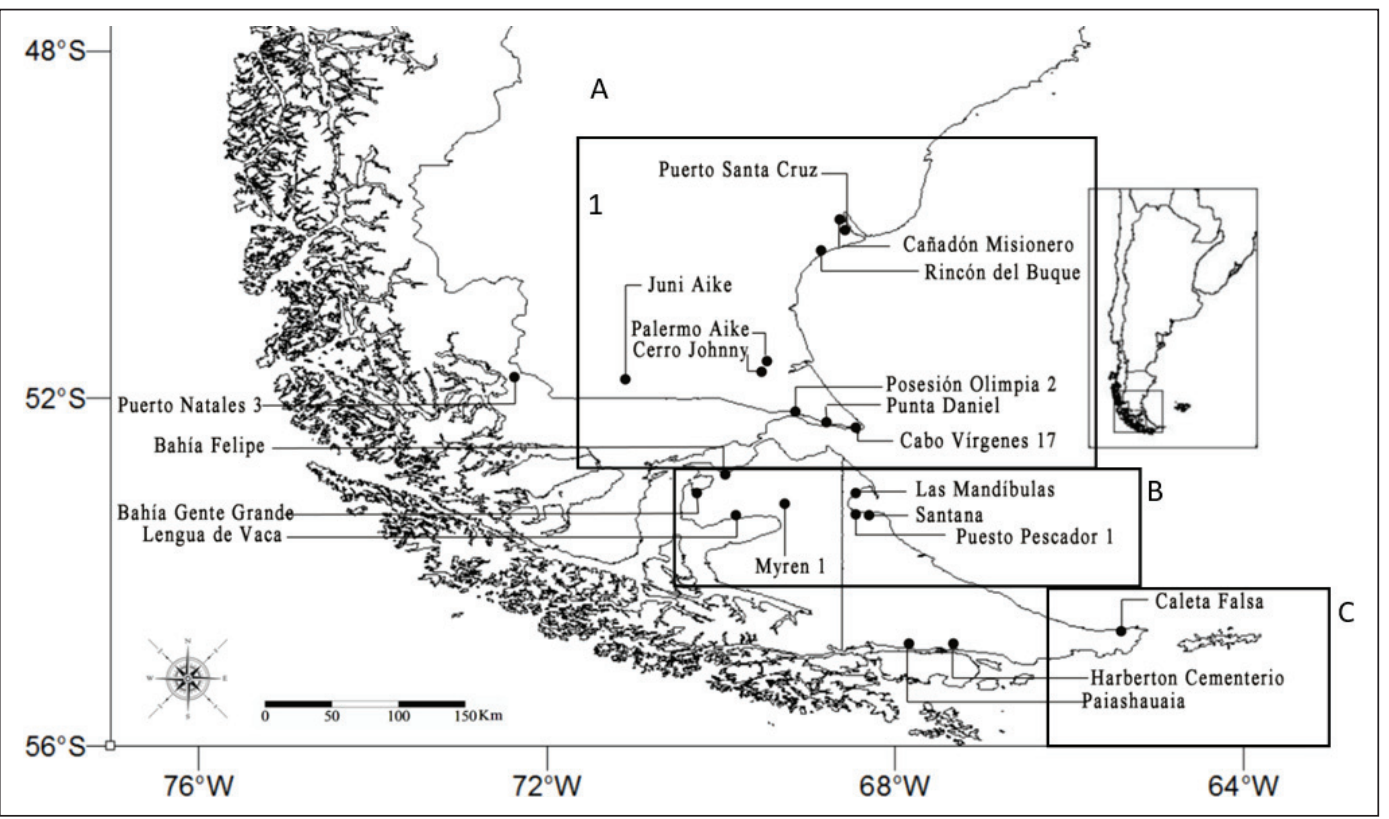

Fig. 1. Localización de los sitios arqueológicos de donde provienen los individuos analizados en este trabajo. Referencia: 1:Sitios del interior delimitados por un circulo; A: Sta Cruz/Magallanes; B: Norte de Tierra del Fuego; C: Sur de Tierra del Fuego. 


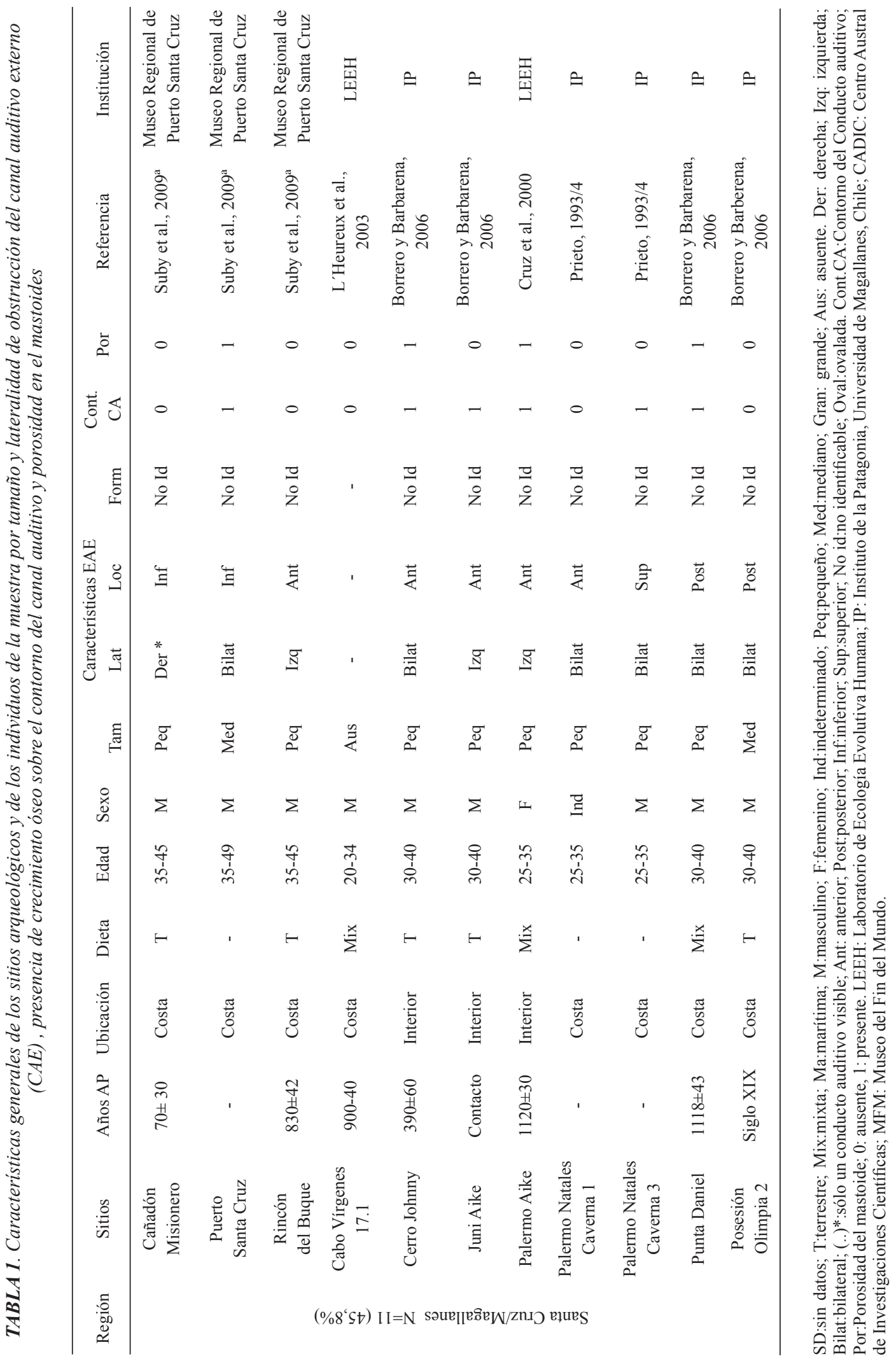




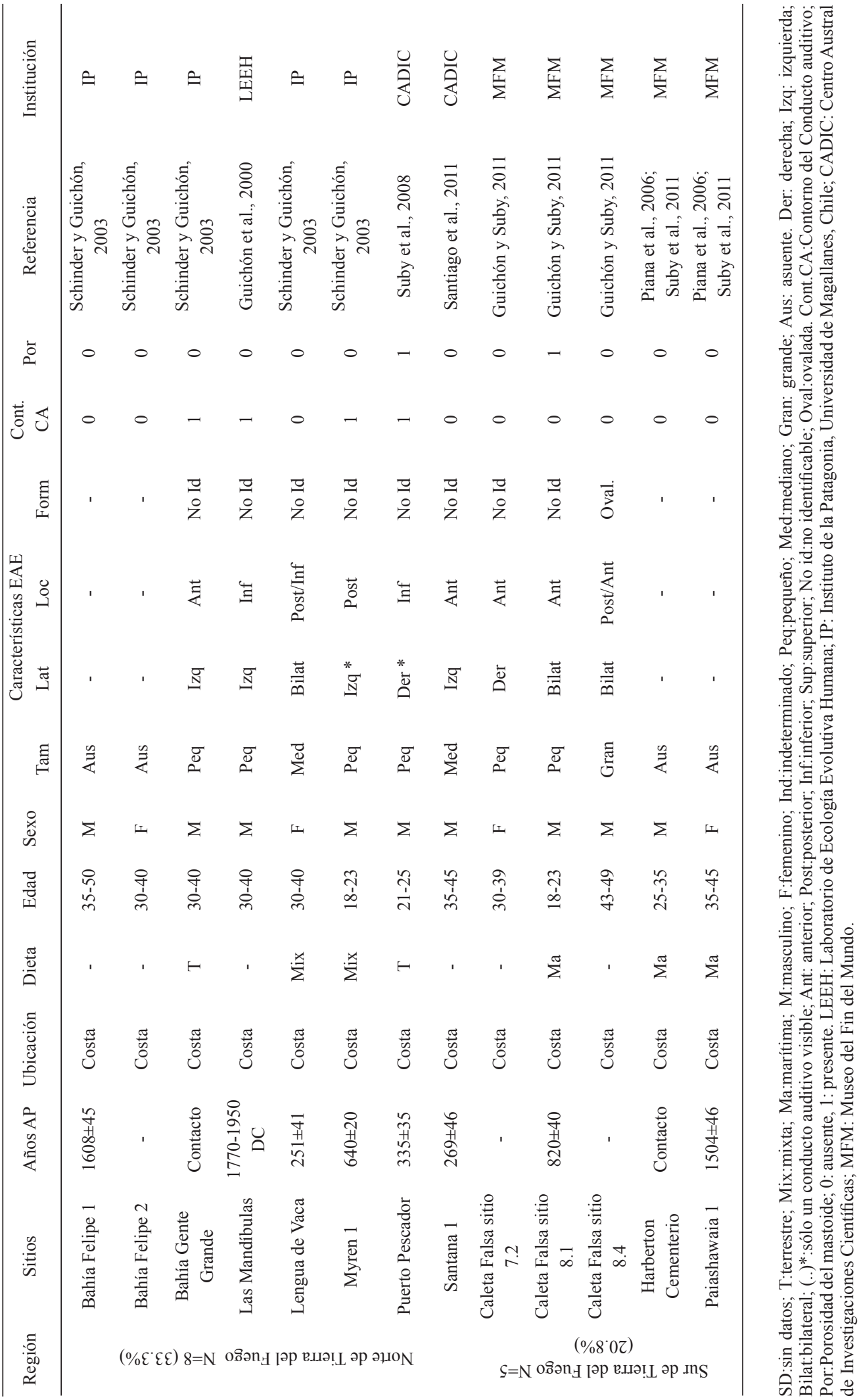


distinguible macroscópicamente del patrón circular normal (Hurst W., Bailey y Hurst B., 2004; Villotte, Stefanovic y Knüsel, 2014). Se diferenció además de los osteomas, los cuales son tumores óseos verdaderos pedunculados de base pequeña, que surgen unilateralmente a lo largo de las suturas tímpano-mastoidea, más externos que las EAE (Graham, 1979; Timofeev et al., 2004). Por lo tanto, la EAE fue diagnosticada cuando las lesiones no presentaron pedúnculos, característicos de los osteomas.

La EAE fue descripta siguiendo la metodología propuesta por Standen et al. (1997), según la cual se relevan cuatro variables: 1- el tamaño: pequeño (bloquea menos que $1 / 3$ del canal); medio (bloquea entre $1 / 3$ y $2 / 3$ ); y grande (bloquea más que 2/3); 2- la localización: pared superior, inferior, anterior o posterior del canal; 3- la forma: esferoidal u ovoidal; y 4- la lateralidad: unilateral o bilateral. Además, se registró la formación de hueso nuevo en el contorno del canal auditivo externo y la porosidad en el proceso mastoides, siguiendo el criterio ausencia (0) o presencia (1), dado que algunas evidencias sugieren que podría estar relacionado con el desarrollo de mastoiditis y eventualmente con modificaciones del conducto auditivo (Flohr y Schultz, 2009; Flohr, Kierdorf y Schultz, 2009).

Se calcularon las frecuencias de EAE para el total de la muestra y posteriormente para submuestras clasificadas según el sexo y la edad de muerte. Los individuos se agruparon en dos categorías etarias: adulto joven (20-35 años) y adulto medio (35-50 años) siguiendo a Buikstra y Ubelaker (1994).

Con el objetivo de explorar la posible relación entre la EAE y las prácticas económicas, se analizó la distribución de las lesiones de acuerdo a la región de procedencia, la proximidad a la costa y el patrón dietario a partir de isotopos estables. En relación con las regiones analizadas, se clasificaron los individuos de acuerdo a su procedencia en Santa Cruz/Magallanes, Norte y Sur de Tierra del Fuego (TDF). En este sentido, el lago Fagnano fue propuesto como referencia física para esta última división de Tierra del Fuego. Se consideraron como sitios "costeros", aquellos que se encuentran a una distancia de $20 \mathrm{~km}$ desde la línea de costa hacia el interior en el área meridional de Patagonia continental, y hasta los $30 \mathrm{~km}$ desde la línea de costa para los sitios de Tierra del Fuego (Barberena, 2008; Borrero y Barberena, 2006; Borrero y Charlin, 2010). Los sitios restantes fueron denominados como "del interior" (Barberena, 2002; Borrero y Barberena, 2006). Por último, la muestra fue clasificada según la dieta en aquellos individuos con consumo predominante de recursos terrestres, marítimos y aquellos que tuvieron una dieta mixta (Tabla 1), en el sentido propuesto en Borrero y Barberena (2006), según el cual no hay un predominio de consumo de un tipo de recurso por sobre el otro. Las diferencias entre grupos fueron analizadas estadísticamente a través del test $F$ de Fisher $(\alpha=0,05$; dos colas $)$.

\section{RESULTADOS}

En la Tabla 1 se presentan los resultados obtenidos para cada uno de los 24 individuos analizados acerca de la presencia o ausencia de EAE, su tamaño, localización, lateralidad y forma, además de la formación de hueso en el contorno del canal auditivo y la porosidad en el hueso temporal. El 79,2\% (19 de 24 individuos) presenta EAE. Se registraron los tres tamaños (Tabla 1; Fig. 2), siendo el pequeño y mediano los más comunes, con $73,7 \%(14 / 19)$ y $21,1 \%$ (4/19) respectivamente. El tamaño grande está presente en solo un individuo adulto medio (5,2\%), proveniente del sitio Caleta Falsa 8.4, localizado en la costa del extremo meridional de Tierra del Fuego (Tabla 1). De los individuos afectados y con ambos CA presentes, el 56,3\% (9/16) presenta EAE bilateralmente (Tabla 1). De los 7 individuos restantes, seis fueron afectados en el CA izquierdo $(85,7 \%)$ y sólo 1 en el CA derecho (14,3\%).

Con respecto a la localización, el 52,6\% (10 de 19) de los individuos con EAE presentó lesiones en la pared anterior del CA, 26,3\% (5/19) en la pared inferior, $26,3 \%(5 / 19)$ en la pared posterior y $5,3 \%(1 / 19)$ en la pared superior. Sin embargo, de los 19 individuos con EAE, uno presentó modificaciones del CA en las paredes anterior y posterior, mientras que en otro se registró en las paredes posterior e inferior. Solo pudo identificarse la forma en uno de los individuos (Caleta Falsa esqueleto 8.1), el único con lesiones de tamaño grande, siendo de forma ovalada. En los restantes, no pudo registrarse esta característica por el reducido tamaño de las 


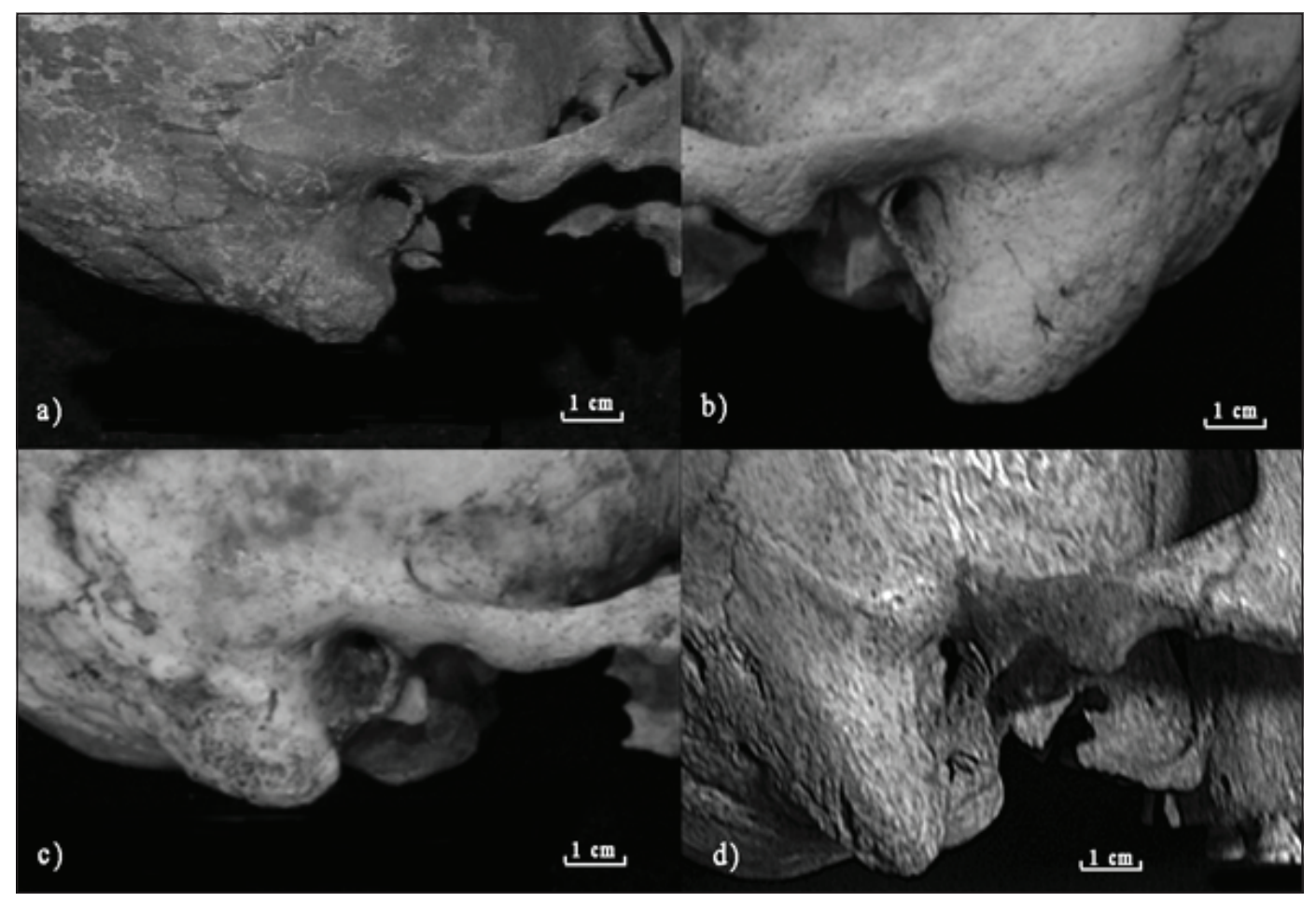

Fig. 2. Tamaños de oclusión de exostosis auditiva externa. a) ausente: conducto normal; b) pequeño: obstrucción hasta 1/3 del conducto; c) mediano: hasta 2/3 de oclusión del conducto auditivo; d) grande: oclusión de más de $2 / 3$ del conducto auditivo.

TABLA 2. Prevalencias de individuos afectados por obstrucción del CA, porosidad y formación de hueso en el contorno del CA

\begin{tabular}{ccccc}
\hline & \multicolumn{4}{c}{ Obstrucción del CA } \\
\hline & $\begin{array}{c}\text { Sin contorno del CA } \\
\text { y porosidad del } \\
\text { mastoides }\end{array}$ & $\begin{array}{c}\text { Con formación ósea } \\
\text { del contorno y } \\
\text { porosidad del mastoides }\end{array}$ & $\begin{array}{c}\text { Con formación } \\
\text { ósea del contorno }\end{array}$ & $\begin{array}{c}\text { Con porosidad } \\
\text { del mastoides }\end{array}$ \\
\cline { 2 - 5 } $\begin{array}{c}\text { Individuos } \\
\text { con EAE }\end{array}$ & $8 / 19(42,1 \%)$ & $5 / 19(26,3 \%)$ & $5 / 19(26,3 \%)$ & $1 / 19(5,3 \%)$ \\
$\begin{array}{c}\text { Individuos } \\
\text { sin EAE }\end{array}$ & $0 / 5(0 \%)$ & $0 / 5(0 \%)$ & $0 / 5(0 \%)$ & $0 / 5(0 \%)$ \\
\hline
\end{tabular}

lesiones.

La formación de hueso nuevo en el contorno del CA afectó al 41,7\% (10 de 24 individuos) del total de la muestra y un 52,6\% (10 de 19 individuos) del total de los individuos que presentaron EAE (Tabla 1). La porosidad del mastoides se registró en un $25 \%(6 / 24)$ sobre el total de los individuos y un 31,6\% (6/19) sobre el to- tal con EAE (Tabla 2). De los 19 individuos con EAE, el $42,1 \%(n=8)$ presentó solo obstrucción del CA, el 26,3\% (5 de 19) obstrucción del CA $\mathrm{y}$ formación de hueso del contorno y porosidad, el 26,3\% de los individuos (5 de 19) mostró obstrucción del CA y formación de hueso y en el 5,3\% (1 de 19) de los individuos se registró la presentó obstrucción del CA y porosidad del 
mastoides (Tabla 2). Ninguno de los individuos sin obstrucción del canal presentó porosidad del mastoides o formación de hueso sobre el contorno del CA (Tabla 2).

Los individuos masculinos presentaron mayor frecuencia de $\operatorname{EAE~}(83,3 \%$; 15 de 18 individuos), que los individuos femeninos (60\%; 3 de 5 individuos). El 75\% (6/8) de los adultos jóvenes (20-35 años) presentaron EAE, porcentaje similar al registrado entre los adultos medios (81,3\%; 13/16) (Fig. 3; Fig. 4). Sin embargo, no se encontraron diferencias estadísticamente significativas cuando se evaluaron las diferencias de prevalencia de EAE en relación al sexo y la edad de muerte ( $\mathrm{p}=0,9$ en ambos casos).

En los individuos provenientes de Santa Cruz/Magallanes y el norte de Tierra del Fuego se registró una mayor prevalencia $(90,9 \%$ y $75 \%$, respectivamente) que los provenientes del sur de Tierra del Fuego (60\%), aunque esta última está representada por un menor número de individuos. Entre los individuos con EAE, el $52,6 \%(10 / 19)$ pertenecen a sitios de Santa Cruz/ Magallanes, el 31,6\% (6/19) pertenecen al norte de TDF y el $15,8 \%$ restante (3/19) al Sur de TDF (Tabla 3). En ningún caso las diferencias entre grupos resultaron significativas (Tabla 3 ).

En relación con el tipo de economía inferido a partir de análisis paleodietario (Barberena, 2002; Santiago, Salemme, Suby y Guichón, 2011; Tessone et al., 2003; Yesner et al., 2003), el $100 \%(7 / 7)$ de los individuos con dietas te-
TABLA 3. Prevalencias de individuos con EAE según el tipo de economía y procedencia

\begin{tabular}{lccc}
\hline & Observaciones & $\mathrm{n}$ & $\%$ \\
\hline Región & & & \\
SC/Mag & 10 & 11 & $90,9(\mathrm{p}=0,9)^{1}$ \\
NTDF & 6 & 8 & $75 ;(\mathrm{p}=0,9)^{2}$ \\
STDF & 3 & 5 & $60(\mathrm{p}=0,7)^{3}$ \\
\hline
\end{tabular}

Economía

Terrestre

$7 \quad 7 \quad 100(\mathrm{p}=0,99)^{4}$

Mixta

4

$580(\mathrm{p}=0,9)^{5}$

Marítima

1

$333,3(\mathrm{p}=0,58)^{6}$

Distancia

a la costa

\begin{tabular}{cccc} 
Interior & 3 & 3 & $100(\mathrm{p}=0,9)^{7}$ \\
Costa & 16 & 21 & 76.2 \\
\hline
\end{tabular}

Probabilidades del análisis estadístico:p1=Sc/Mag vs. STDF; $\mathrm{p} 2=\mathrm{SC} / \mathrm{MAG}$ vs. NTDF; $\mathrm{p} 3=\mathrm{NTDF}$ vs. STDF; $\mathrm{p} 4=$ Terrestre vs. Mixta; 5 =Mixta vs. Marítima; 6 =Terrestre vs. Marítima; p7=interior vs. Costa.

rrestres presentaron EAE. Por su parte, el $80 \%$ (4/5) de los individuos con dietas mixtas estuvieron afectados, mientras solo se detectó EAE en el $33,3 \%(1 / 3)$ de los individuos con dietas

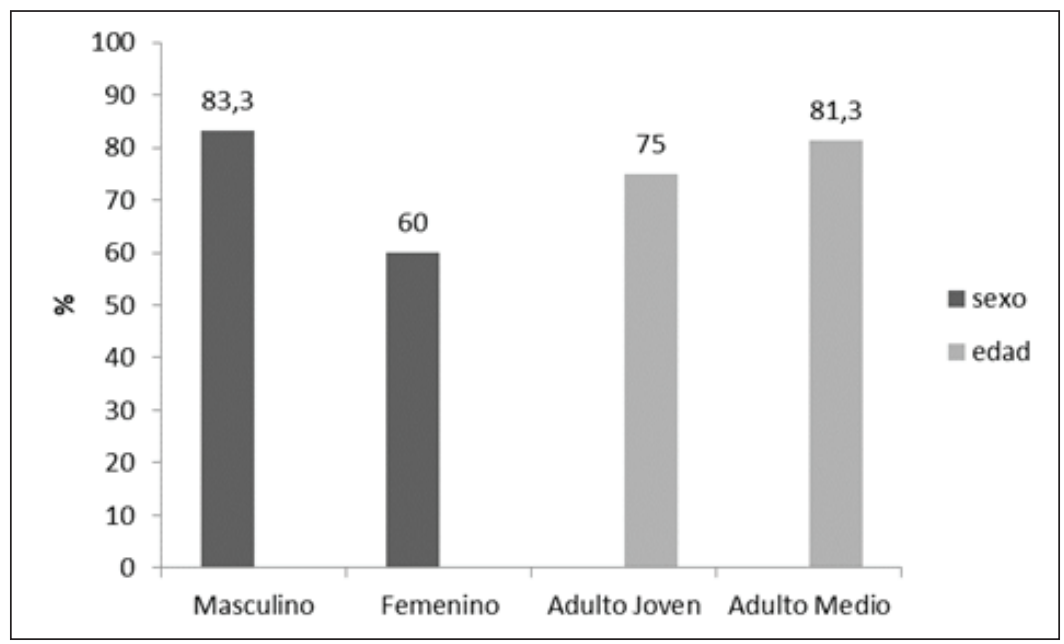

Fig. 3. Prevalencias de individuos con EAE según el sexo y el rango etario (\%). 


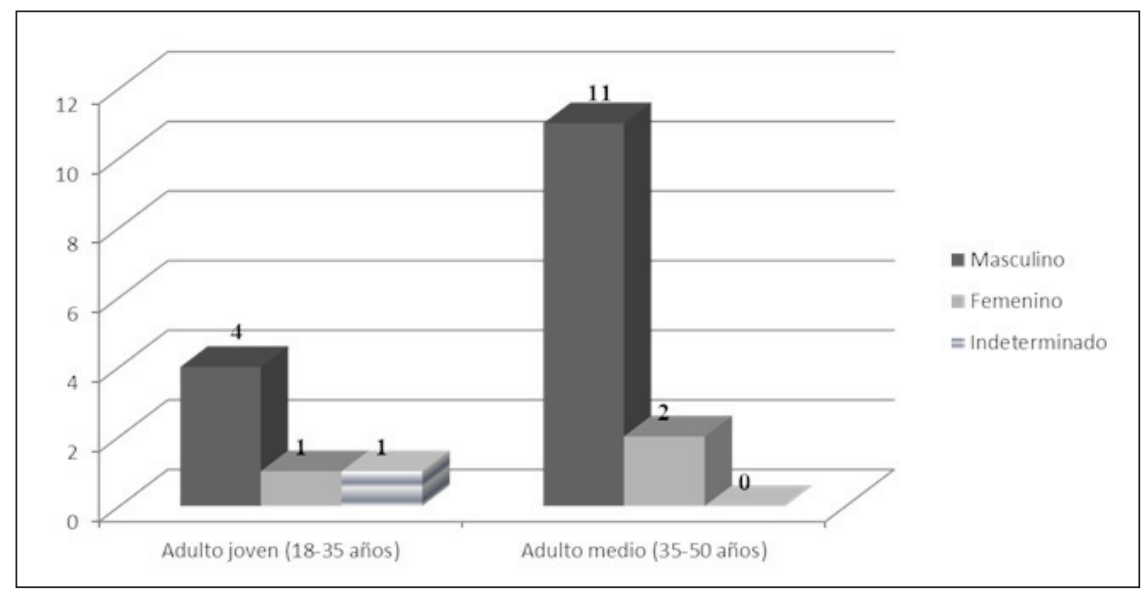

Fig. 4. Prevalencias de individuos con EAE según el sexo y el rango etario (n afectados).

marítimas (Tabla 3). Nuevamente, las diferencias entre grupos no resultaron estadísticamente significativas (Tabla 3).

En relación con la proximidad a la costa, el $76,2 \%(16 / 21)$ de los individuos recuperados en sitios costeros presentaron EAE, mientras que el 100\% (3/3) de los individuos de sitios del interior mostraron este rasgo (Tabla 3 ). El 55,6\% $(5 / 9)$ de los restos recobrados en sitios costeros tenía una economía de subsistencia basados en su predominancia en recursos terrestres, en el $33,3 \%(3 / 9)$ la dieta era mixta y en el $11,1 \%$ $(1 / 9)$ predominaba una economía en base a recursos marinos (Tabla 1). En este caso ninguna de las categorías establecidas para la distancia a la costa resultó estadísticamente significativa (Tabla 3).

\section{DISCUSIÓN}

El estudio de la EAE ha sido empleado en las últimas décadas como una manera de interpretar el impacto de las economías ligadas a las prácticas extractivas marítimas sobre la salud. La hipótesis hidrotérmica propuesta por Kennedy (1986) es comúnmente considerada como una de las causas más frecuentes del desarrollo de la EAE en poblaciones del pasado, en especial en grupos costeros entre los $30^{\circ}$ y $45^{\circ}$ de latitud Norte y Sur. Sin embargo, esta hipótesis no ofrece explicaciones acerca de cuáles son los procesos involucrados en el desarrollo de la EAE en latitudes superiores o en algunas pobla- ciones sin acceso a la costa (e.g. Okumura et al., 2007; Standen et al., 1997). La incorporación de otro tipo de variables, ambientales y biológicas, permite explorar la presencia de EAE en restos óseos en circunstancias donde la inmersión en aguas de baja temperatura resulta poco probable por el riesgo a sufrir hipotermia y en aquellas poblaciones con hábitos terrestres no asociados a los ambientes acuáticos. Los resultados presentados en este trabajo constituyen un paso siguiente al primer esfuerzo presentado por Ponce et al. (2008) por probar esta hipótesis en restos humanos provenientes de Patagonia Austral.

Las prevalencias de EAE en muestras de individuos del pasado son variables. La Tabla 4 sintetiza los principales estudios publicados recientemente sobre EAE, registrada en individuos de Sudamérica, Norte y Sur de Europa, Norte de África (i.e. Egipto), Medio Oriente y Eurasia oriental (i.e. China). Esta distribución geográfica puede estar influenciada con el mayor número de estudio paleopatológicos realizados en algunas de ellas. Las evidencias más antiguas datan de cerca de 10 mil años AP en Anatolia (i.e. Turquía), mientras que no son frecuentes los estudios en muestras con cronologías más recientes. Al comparar los resultados obtenidos en este trabajo con los antecedentes sintetizados en la Tabla 4, se observa una mayor prevalencia de EAE en la muestra analizada en comparación a los porcentajes registrados en individuos de otras poblaciones que habitaron latitudes mayores a $45^{\circ} \mathrm{N}$ y S (e.g. Ponce et al., 2008; Villotte 


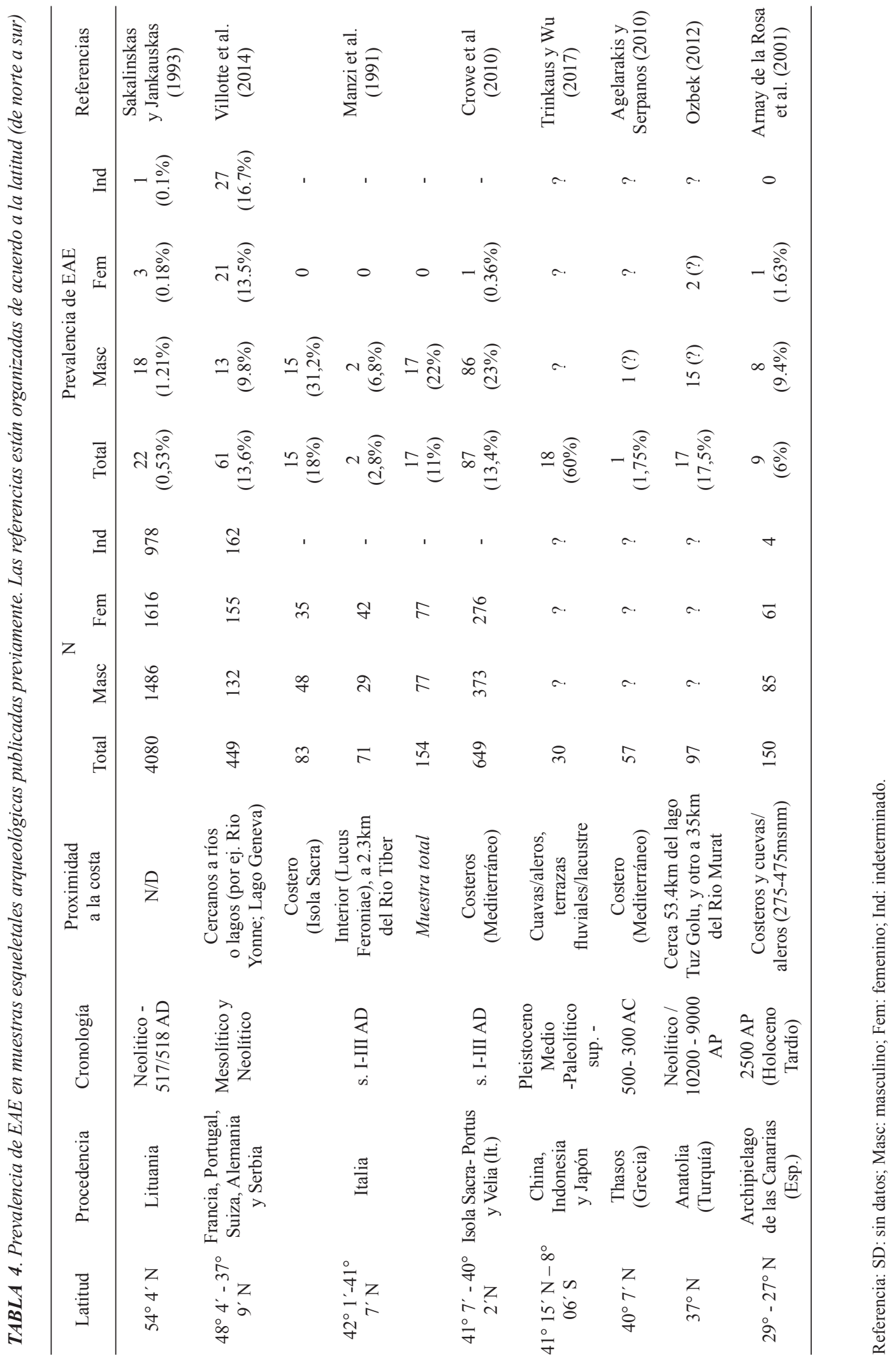




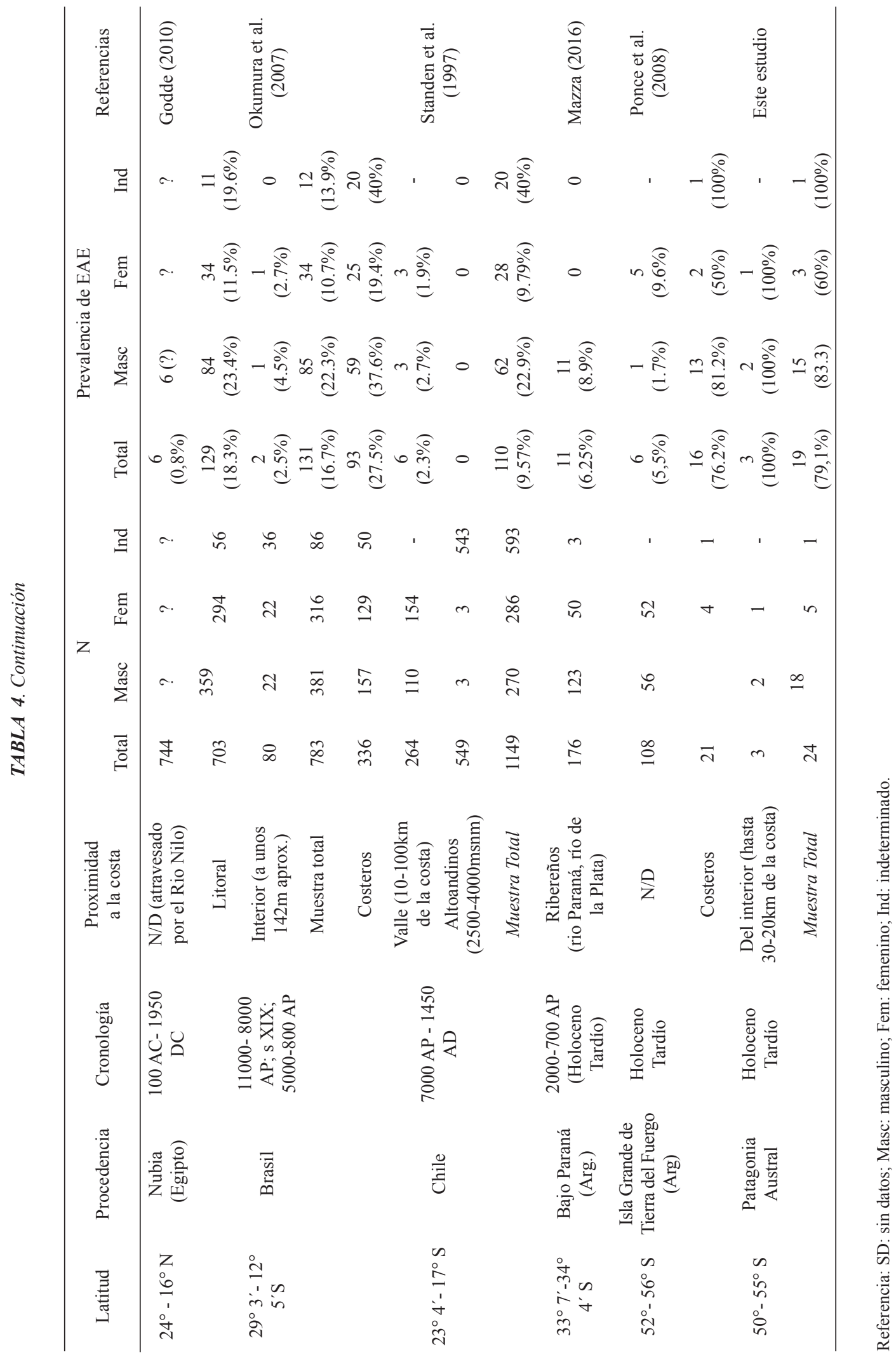


et al., 2014). Incluso el porcentaje de individuos con EAE es mayor en comparación con poblaciones que habitaron entre los $30^{\circ}$ y $45^{\circ}$ (Crowe et al., 2010; Manzi, Aperduti y Passarello, 1991; Mazza, 2016; Ozbek, 2012). Por último, Hrdlicka (1935) observó un $0.2 \%$ de EAE en 1000 cráneos pertenecientes a una colección de restos de esquimales (Inuit). Sin embargo, esta última referencia no indica la procedencia y la cronología de la muestra por lo que no ha sido agregada a la Tabla 4.

La prevalencia total de EAE en Patagonia Austral es más próxima a la muestra analizada en Eurasia oriental, con $60 \%$ sobre individuos del Pleistoceno (medio y superior) y Paleolítico (Trinkaus y Wu, 2017), y a las informadas en muestras de individuos actuales que practican deportes acuáticos como el surf (Kroon et al., 2002; Umeda, Nakajima y Yoshioka, 1989). Por lo tanto, la muestra analizada presenta una de las prevalencias más altas registradas en restos arqueológicos hasta el momento. Estos resultados contrastan con los reportados previamente por Ponce et al. (2008), posiblemente debido a diferencias en las metodologías de registro y las muestras empleadas, por lo que comparaciones directas entre ambos estudios no resultan posibles.

Respecto al sexo, la edad y la distribución y tipo de lesiones y asociada a la práctica económica los resultados no mostraron diferencias estadísticamente significativas, posiblemente debido al reducido tamaño muestral y al amplio rango cronológico. Sin embargo, es posible señalar algunas tendencias que podrían sugerir hipótesis a ser evaluadas en el futuro.

Los individuos masculinos mostraron una mayor prevalencia de EAE que las mujeres, al igual que en la mayoría de los estudios previos (Arnay de la Rosa et al., 2001; Castro et al., 2016; Crowe et al., 2010; Goode, 2009; Standen et al., 1997; Tabla 4), incluso con diferencias estadísticamente significativas (Mazza, 2016). Por el contrario, Villotte et al. (2014) mostraron una mayor prevalencia en mujeres $(13,5 \%)$ que en hombres $(9,8 \%)$ en una muestra de restos de europeos, aunque sin diferencias significativas. En las poblaciones de Patagonia Austral, las prácticas sociales diferenciales entre los sexos podrían estar involucradas en el mayor porcentaje de EAE registrado en los individuos mas- culinos, aunque por el momento no existen evidencias etnográficas claras que puedan sostener esta hipótesis.

La EAE es una condición progresiva, es decir que requiere de la acción de los mecanismos involucrados durante un tiempo prolongado, lo que parecería reflejar su ausencia en individuos sub-adultos (Crowe et al., 2010; Deleyiannis et al., 1996; DiBartolomeo, 1979; Kroon et al., 2002). Por el contrario, suele producirse con mayor frecuencia con el aumento de la edad (Deleyiannis et al., 1996; Standen et al., 1997). Los resultados presentados en este trabajo acompañan esta tendencia, indicando que los adultos medios presentaron una prevalencia levemente mayor de EAE que los adultos jóvenes, aunque sin presentar diferencias significativas. En esta dirección, el único caso con un desarrollo de la EAE de tamaño grande junto con los cuatro casos con EAE de tamaño mediano se registraron en individuos adultos medios, todos ellos procedentes de sitios costeros. Por el contrario, en la mayoría de los individuos jóvenes que presentaron EAE las lesiones fueron identificadas de tamaño pequeño. Aunque parece existir una tendencia en favor de los individuos adultos medios, no es posible establecer con certeza que las lesiones identificadas no hayan comenzado a desarrollarse durante la juventud. Al mismo tiempo, los resultados pueden estar también influidos por los sesgos metodológicos en la estimación de la edad.

Respecto de la distribución de las lesiones, se observó un predominio del desarrollo de la EAE bilateral sobre los individuos con los dos CA conservados, lo que sigue algunas de las propuestas previas (e.g. Agelarakis y Serpanos, 2010; Mazza, 2016). Sin embargo, Trinkaus y Wu (2017) encontraron una mayor tendencia de EAE unilateral sobre su muestra. Por otro lado, la mayor parte de los individuos mostró formación de hueso en la porción anterior del CA, seguido por la porción inferior y posterior. Solo un reducido porcentaje presentó obstrucción de la pared superior del CA. Se ha sugerido que el análisis de la localización de la EAE en el $\mathrm{CA}$, junto con la forma, es un medio para "evaluar si la EAE es parte de la placa timpánica o un fenómeno independiente" (Standen et al., 1997: 123). Si bien diversos trabajos registraron su localización (Arnay de la Rosa et al., 2001; 
Crowe et al., 2010; Mazza, 2016; Standen et al., 1997; Velasco-Vazquez et al., 2000; Villotte et al., 2014), en general no incorporan su análisis en los resultados y conclusiones de sus trabajos. Mazza (2016) menciona que todas las EAE $(\mathrm{n}=11)$ se encuentran en una posición anterior en el CA, Velasco-Vázquez et al. (2000) observaron que en la mayoría de los casos estaban localizados en la pared postero-inferior (75.51\%) $y$ antero-superior $(22.44 \%)$, mientras que Trinkaus y Wu (2017) y Standen et al. (1997) observaron que se ubican en la parte posterior en su mayoría. Es decir, la posición de la EAE es una característica variable que podría depender, en parte, de la susceptibilidad local de los tejidos circundantes del CA, por lo que parece no tener demasiado significado patológico, sino más bien descriptivo.

La EAE está presente tanto en individuos asociados a poblaciones con economía marina como en aquellas con economía terrestre. De hecho, la mayor frecuencia fue observada entre los individuos asociados a dietas terrestres y mixtas, tanto en contextos costeros como interiores. A su vez, resultó más frecuente entre los individuos de la región de Santa Cruz/Magallanes que en el norte y sur de Tierra del Fuego. Estos resultados no permitirían explicar la inmersión en agua como agente causal de la EAE en individuos con dietas terrestres, incluyendo aquellos recuperados en sitios del interior. Sin embargo, las evidencias acerca de los patrones de movilidad y el uso de los ambientes por parte de los individuos (e.g. Borrero, Barberena, Franco, Charlin y Tykot, 2009) podrían implicar contactos esporádicos de ambientes marítimos por parte de grupos con hábitos terrestres. Por lo tanto, no es posible rechazar por completo que al menos algunos de los individuos recuperados en Santa Cruz/Magallanes que en el norte y sur de Tierra del Fuego, más asociados a dietas terrestres y mixtas, hayan tenido algún vínculo con la costa y el uso del agua que pudiera haber influido en el desarrollo de la EAE.

Aunque las diferencias entre las prevalencias de acuerdo a la proximidad a la costa, la región y la economía no resultaron significativamente diferentes en ningún caso (Tabla 3), los resultados parecen sugerir que el desarrollo de la EAE podría no estar completamente vinculado al contacto con el agua en las poblaciones de Patago- nia Austral, a pesar de que la prevalencia hallada es próxima a la observada en quienes practican deportes acuáticos en la actualidad. Las fuentes etnográficas y etnohistóricas no mencionan la práctica del buceo o sumergimiento como un tipo de recolección durante periodos históricos (e.g. Gusinde, 1986; Orquera y Piana, 1999; Saletta, 2014), y se desconoce su empleo en periodos previos. Tampoco se conocen evidencias de buceo en cursos de agua interiores por parte de las poblaciones vinculadas a prácticas terrestres, aunque esta actividad no puede ser desestimada por completo, al menos ocasionalmente. En este sentido, las mayores prevalencias en grupos con economías terrestres podrían indicar una mayor influencia de otros factores causales de la EAE en estas poblaciones.

Incluso en el caso de los individuos asociados a dietas marítimas, la inmersión en agua podría no ser el único factor involucrado en el desarrollo de la EAE. Estudios actuales muestran que el proceso de hipotermia comienza en aguas por debajo de los $11^{\circ} \mathrm{C}$, como las registradas en el Atlántico Sur, produciendo que la temperatura corporal descienda por debajo de $\operatorname{los} 35^{\circ} \mathrm{C}$ y resultando mortal luego de 45-60 minutos de exposición (Molnar, 1946; Keatinge, Khartchenko, Lando y Lioutov, 2001), por lo que no resulta esperable este tipo de prácticas por periodos prolongados.

En base a estos resultados, si bien no es posible descartar por completo que el contacto con el agua haya influido en el desarrollo de EAE en estos individuos, no permite explicar la prevalencia similar encontrada en otros contextos asociados a economías terrestres y en sitios del interior de Patagonia Austral. Por el contrario, la posible combinación con otros factores, como el viento y la baja temperatura ambiental, podrían explicar la presencia de EAE en individuos con economías terrestres y recuperados en sitios del interior (Villotte y Knüsel, 2015). Sumado a la temperatura ambiental, la acción del viento Oeste/Sudoeste como un factor constante y con velocidades que pueden llegar hasta los $100 \mathrm{~km} / \mathrm{h}$ durante algunos días del año (Goode, 2009), este tipo de ambiente pudo haber actuado de modo refrigerante sobre el conducto auditivo externo. Dicha acción podría desencadenar procesos irritativos e incluso inflamatorios (por ejemplo, otitis) en el epitelio del conducto audi- 
tivo (House y Wilkinson, 2008; Hutchinson et al., 1997; Timofeev et al., 2004). Si estos procesos continúan con el tiempo, es posible esperar que se desencadene la actividad osteoblástica que llevaría al desarrollo de EAE. Sin embargo, estas hipótesis deben ser fortalecidas en el futuro mediante el análisis de muestras de mayor tamaño, particularmente con mayor número de individuos del interior y con dietas marítimas que las analizadas en este trabajo.

Desde un punto de vista metodológico, el estudio de la EAE ha sido realizado en restos humanos mediante dos tipos diferentes de método de registro (Crowe et al., 2010; Standen et al., 1997), lo que en principio dificulta la comparación de resultados. Sin embargo, ambos métodos presentan criterios idénticos en cuanto al desarrollo de las lesiones del CA, característica importante ya que condiciona los síntomas que padecerán los individuos, como por ejemplo la pérdida de audición. La metodología de Standen et al. (1997) aplicada en este estudio permite evaluar la localización y la forma de la EAE, resultando de utilidad en aquellos individuos que presentan un tamaño grande de EAE en donde la variable forma (sea esferoidal u ovoidal) resulta distinguible, al contrario, en aquellos con tamaños pequeños o medianos. Sin embargo, no fue posible distinguir este aspecto, salvo en el individuo del sitio Caleta Falsa esqueleto 8.4, el cual presentó una EAE de tamaño grande y que se distingue este rasgo de forma ovalada sobre la pared posterior y anterior del conducto auditivo (Fig. 2d).

Finalmente, la mayoría de las lesiones registradas en los casos de EAE, presentaron obstrucción del CA sin ningún otro tipo de lesión. Sin embargo, la formación de hueso en el contorno se presenta acompañando a la obstrucción del CA en un $26,3 \%$ (5/19), por lo que no puede descartarse que pueda estar vinculada a su desarrollo. Por el contrario, menos frecuente fue la porosidad del mastoides. Estos resultados por el momento no permiten distinguir una propuesta clara que brinde información acerca de la naturaleza infecciosa de la exostosis o patologías afines, como la otitis media crónica. Sin embargo, ambas modificaciones morfológicas solo se presentaron en aquellos individuos con EAE, ya sea de manera conjunta o independiente, lo que podría indicar alguna vinculación con esta patología. Por este motivo es necesarioavanzar sobre esta posible relación y sus significados patológicos (Macías, Villanueva y Ruzapérez-Barquero, 1999; Malard, Beauvillain de Montreuil y Legent, 2005). En este sentido, por ejemplo, las irritaciones crónicas generalizadas en el pabellón auricular afectan de manera conjunta al oído externo, oído medio, apófisis mastoidea e incluso a la articulación temporomandibular y a la primera porción del tracto respiratorio (a través de la Trompa de Eustaquio) (Campos, Barrón y Fajardo, 2014; Malard et al., 2005). Además, la otitis media crónica u otitis externa maligna pueden presentar diseminación de pus frecuentemente hacia las celdillas mastoideas generando erosión en las mismas (Campos et al., 2014; Kahl y Nickol, 1977).

\section{CONCLUSIONES}

Los estudios de la exostosis auricular externa (EAE) permiten no solo conocer aspectos asociados con la salud de las poblaciones humanas, sino también su vinculación con el ambiente y con el comportamiento sociocultural expresado el desarrollo de prácticas económicas como el aprovechamiento de recursos acuáticos. Los resultados de este trabajo presentan una alta prevalencia de EAE en las poblaciones humanas de Patagonia Austral, mayor a la reportada anteriormente, en general de reducido tamaño y bilateral y con mayor frecuencia en hombres que en mujeres. Aunque este trabajo forma parte de un acercamiento a esta problemática, debe ser continuada en los próximos años, discutiendo sus alcances bajo evidencias logradas a partir de conjuntos de esqueletos más numerosos y su comparación con resultados que serán obtenidos en restos humanos provenientes de otras poblaciones costeras y no costeras. Por otro lado, la amplitud cronológica de la muestra analizada podría suponer modificaciones en la actividad desarrollada durante ese rango de tiempo. Por eso, conjuntos más acotados temporalmente podrían brindar información con mayor detalle.

La alta prevalencia de EAE registrada en individuos asociados a economías terrestres observadas en este trabajo permite sugerir que la inmersión en agua no sería el único factor involucrado en su desarrollo. Al mismo tiempo, debido a las bajas temperaturas registradas en el Atlántico Sur, por debajo de los 10 grados centígrados durante 
todo el año, no es esperable que la práctica del buceo haya sido frecuente y sostenida. Sin embargo, la prevalencia total de EAE en esta muestra particular de individuos de Patagonia Austral resultó más próxima a las informadas en muestras actuales de personas que practican deportes acuáticos. Si bien la EAE en general fue tenida en cuenta como un indicador de actividades de subsistencia marina de los grupos prehistóricos, los resultados hallados en este trabajo permiten asumir que otros factores ambientales, como el viento, la humedad y las bajas temperaturas, pueden ser igualmente importantes, facilitando su desarrollo en elevadas frecuencias.

\section{AGRADECIMIENTOS}

Agradecemos a los organismos científicos que financiaron el trabajo. A los Dres. Luis Borrero, Ricardo Guichón, Mónica Salemme, Fernando Santiago, Ernesto Piana y Francisco Zangrando por permitir el acceso a los restos recuperados en el marco de sus respectivos proyectos de investigación. Finalmente agradecemos a los dos revisores anónimos y a los editores por las sugerencias brindadas durante el desarrollo del mismo.

\section{LITERATURA CITADA}

Administración de Parques Nacionales. (2007). Plan De Manejo. Parque Nacional Tierra del Fuego. Recuperado en https://www.sib.gov.ar/archivos/PM_TIERRA_ DEL_FUEGO.pdf

Agelarakis, A., y Serpanos, Y. C. (2010). Auditory exostoses, infracranial skeletomuscular changes and maritime activities in classical period thasos island. Mediterranean Archaeology and Archaeometry, 10 (2), 45-57.

Arnay de la Rosa, M., Velasco-Vazquez, J., Gonzalez Reimers, E., y Santolaria-Fernandez, F. (2001). Auricular exostoses among the prehistoric population of different islands of the Canary archipelago. Annals of Otology, Rhinology and Laryngology, 110, 1080-1083.

Azizi, M. H. (2011). Ear Disorders in Scuba Divers. International Journal of Occupational and Environmental Medicine, 2(1), 20-26.

Bailey, R. G. (1989). Explanatory supplement to ecoregions map of the continents. Environ Conserv, 16(4), $307-$ 309.

Barberena, R. (2002). Los límites del mar. Buenos Aires, Argentina: Sociedad Argentina de Antropología.

Barberena, R. (2008). Arqueología y Biogeografia Humana en Patagonia Meridional. Buenos Aires, Argentina: Sociedad Argentina de Antropología.

Berry, A., y Berry, R. (1967). Epigenetic variation in the human cranium. Journal of Anatomy, 101, 361-379.

Borella, F. (2010). Revisando la interpretación de los restos de lobos marinos en el registro arqueológico. El caso de Cabo Vírgenes (Patagonia meridional). En L. Borrero y
J. Charlin. (Ed.), Arqueología de Pali Aike y Cabo Virgenes (Santa Cruz, Argentina) (pp. 123-155). Buenos Aires, Argentina: Dunken.

Borla, M. L., y Vereda, M. (2015). Explorando Tierra del Fuego: manual del viajero en el fin del mundo. Ushuaia, Argentina: Editorial Utopías. Recuperado en http:// www.tierradelfuego.org.ar/infogeneral/clima

Borrero, L. A., y Barberena, R. (2006). Hunter-gatherer home ranges and marine resources. An archaeological case from Southern Patagonia. Current Anthropology 47(5), 855-867.

Borrero, L. A., y Charlín, J. (2010). Arqueología del campo volcánico Pali Aike, Argentina. En L. Borrero, y J Charlin. (Ed.), Arqueología de Pali Aike y Cabo Virgenes (pp. 9-30). Buenos Aires, Argentina: Dunken.

Borrero, L. A, Barberena, R., Franco, N. V., Charlin, J., y Tykot, R. H. (2009). Isotopes and Rocks: Geographical Organisation of Southern Patagonian Hunter-Gatherers. International Journal of Osteoarchaeology, 19, 309327. doi:https://doi.org/10.1002/oa.1036

Buikstra, J., y Ubelaker, D. (1994). Standards for data collection from human skeletal remains. Fayetteville, AK: Arkansas. Archaeological Survey, Research Series No 44.

Campos, L., Barrón, M., y Fajardo, G. (2014). Otitis media aguda y crónica, una enfermedad frecuente y evitable. Revista de la Facultad de Medicina de la UNAM, 57(1), 5-14.

Castro, M., Goycoolea, M., y Silva-Pinto, V. (2016). External ear canal exostosis and otitis media in temporal bones of prehistoric and historic chilean populations. A paleopathological and paleoepidemiological study. Acta Oto-Laryngologica, 137(4), 365-369. doi:10.1080/0001 6489.2016.1249949

Crowe, F., Sperduti, A., O'Connell, T. C., Craig, O. E., Kirsanow, K., Germoni, P., Macchiarelli, R., Garnsey, P. y Bondioli, L. (2010). Water-related occupations and diet in two Roman coastal communities (Italy, first to third century $\mathrm{AD}$ ): correlation between stable carbon and nitrogen isotope values and auricular exostosis prevalence. American Journal of Physical Anthropology, 142, 355-366.

Cruz, I., Muñoz, A., y Zangrando, A. (2007). La interpretación de los restos de animales pequeños en la arqueología patagónica: estado de la cuestión y perspectivas. En F. Morello, M. Martinic, A. Prieto y G. Bahamonde. (Ed.), Arqueología de Fuego-Patagonia. Levantando piedras, desenterrando huesos... y develando arcanos (pp.15-22). Punta Arenas, Chile: Ediciones CEQUA.

Cruz, I., Astete, F., Nauto, G., y Borrero, L. (2010). La colonia de nidificación de pingüinos de Magallanes de Cabo Vírgenes a lo largo del tiempo. En L. Borrero y J. Charlin. (Ed.), Arqueología de Pali Aike y Cabo Virgenes (Santa Cruz, Argentina) (pp. 137-145). Buenos Aires, Argentina: Dunken.

Deleyiannis, F. W. B., Cockcroft, B. D., y Pinczower, E. F. (1996). Exostoses of the external auditory canal in oregon surfers. American Journal of Otolaryngology Head and Neck Medicine and Surgery, 17(5), 303-307. doi:https://doi.org/10.1016/S0196-0709(96)90015-0

DiBartolomeo, J. R. (1979). Exostoses of the external auditory canal. Annals of Otology, Rhinology \& Laryngology, 88(6), 2-20. doi:https://doi.org/10.2298/ STA $1060137 \mathrm{M}$

Flohr, S., y Schultz, M. (2009). Mastoiditis: Paleopathological evidence of a rarely reported disease. American Journal of Physical Anthropology, 138, 266-273.

Flohr, S., Kierdorf, U., y Schultz, M. (2009). Differential 
diagnosis of mastoid hypocellularity in human skeletal remains. American Journal of Physical Anthropology, $140,442-453$.

Fowler, W., y Osmun, P. (1942). New bone growth due to cold water in the ear. Archeaology Otolaryngology, 36, 455-466.

Garreaud, R. D., Vuille, M., Compagnucci, R., y Marengo, J. (2008). Present-day South American climate. Palaeogeography, Palaeoclimatology and Palaeoecology, 281, 180-195. doi:http://doi.org/10.1016/j.paleo.2007.10.032

Gonzalez Reimers, E., Lorenzo de la Peña, L., SarmientoHerrera, R., Perez Piñero, B., y Arnay de la Rosa, M. (2008). Exotosis auriculares: una lesión del presente y del pasado. Imagen en Osteología, 17(5), 112-113.

Goode, K. (2009). An examination of proposed causes of auditory exostoses. International Journal of Osteoarchaeology. Recuperado en http://www.interscience. wiley.com

Graham, H. (1979). Osteomatas and exostoses of the external auditory canal. Annals of Otology, Rhinology and Laryngology, 88, 566-572.

Gusinde, M. ([1931]1982). Los indios de Tierra del Fuego. Los Selk'nam. I y II. Buenos Aires, Argentina: Centro Argentino de Etnología Americana

Gusinde, M. ([1937]1986). Los indios de Tierra del Fuego. Los Yamanas. I-II-III. Buenos Aires, Argentina: Centro Argentino de Etnología Americana.

Hanihara, T., Ishida, H., y Dodo, Y. (2003). Characterization of biological diversity through analysis of discrete cranial traits. American Journal of Physical Anthropology, 121(3), 241-251. https://doi.org/10.1002/ajpa.10233

Harrison, D. (1951). Exostoses of the external auditory meatus. Journal of Laryngology and Otology, 65,704-714.

House, J. W., y Wilkinson, E. P. (2008). External auditory exostoses: Evaluation and Treatment. Research. Otolaryngology-Head and Neck Surgery, 138, 672-678.

Hrdlicka, L. (1935). Ear exostoses. Nature, 127, 512-513.

Hurst, W., Bailey, M., y Hurst, B. (2004). Prevalence of external auditory canal exostoses in Australian surfboard riders. Journal of Laryngology and Otology, 118, 348351.

Hutchinson, D. L., Denise, C. B., Daniel, H. J., y Kalmus, G.W. (1997). A reevaluation of the cold water etiology of external auditory exostoses. American Journal of Physical Anthropology, 103, 417-422.

Kahl, H., y Nickol, H. J. (1977). Otitis externa maligna. Archives of Oto-Rhino-Laryngology, 216(2), 516-517. https://doi.org/10.1007/BF00458938

Kennedy, G. E. (1986). The relationship between auditory exostoses and cold water: A latitudinal analysis. American Journal of Physical Anthropology, 71(4), 401-415. https://doi.org/10.1002/ajpa.1330710403

Keatinge, W.R., Khartchenko, M., Lando, N., y Lioutov, V. (2001). Hypothermia during sports swimming in water below $11^{\circ} \mathrm{C}$. British Journal of Sports Medicine, 35, 352-353

Kroon, D. F., Lawson, M. L., Derkay, C. S., Hoffmann, K., y McCook, J. (2002). Surfer's ear: External auditory exostoses are more prevalent in cold water surfers. Otolaryngology-Head and Neck Surgery, 126(5), 499-504. https://doi.org/10.1067/mhn.2002.124474

Macías, M., Villanueva, A., Mateo, A., y Ruzapérez-Barquero, M. (1999). Enfermedades otológicas halladas en una muestra de población púnica y romana de Cádiz. Actas del V Congreso Nacional de Paleopatología (Alcalá La Real, 1999), (3), 1-10.

Malard, O., Beauvillain de Montreuil, C., y Legent, F.
(2005). Enfermedades adquiridas del oído externo. EMC-Otorrinolaringología, 34(4), 1-21. doi:https:// doi.org/10.1016/S1632-3475(05)45015-8

Mancini, M.V. (2003). Paleoecología del Cuaternario tardío en el sur de la Patagonia (46-52 S), Argentina. Revista del Museo Argentino de Ciencias Naturales 5, 273-283.

Mancini, M.V., Prieto, A. R., Paez, M. M., y Schábitz, F. (2008). Late Quaternary vegetation and climate of Patagonia. Developments In Quaternary Sciences, 11, 351367.

Manzi, G., Aperduti, A., y Passarello, P. (1991). Behavior induced auditory exostoses in imperial Roman society: evidence from coeval urban and rural communities near Rome. American Journal of Physical Anthropology, 85: 253-260.

Mazza, B. (2016). Auditory exostoses in pre-Hispanic populations of the Lower Paraná Wetlands, Argentina. International Journal of Osteoarchaeology, 26(3), 420-430. doi:https://doi.org/10.1002/oa.2432

McCulloch, R., Bentley, M., Tipping, R., y Clapperton, C. (2005). Evidence for late-glacial ice dammed lakes in the central Strait of Magellan and Bahía Inútil, southernmost South America. Geography Annals, 87(2), 335362 .

Molnar, G.B. (1946). Survival of Hypotermia by men immersed in the ocean. Journal of the American Medical Association, 131(13), 1046-1050. doi:https://doi. org/10.1001/jama.1946.02870300014004

Muñoz, A. (2011). Pinniped zooarchaeological studies in Southern Patagonia: current issues and future research agenda. En N. Bicho, J. Haws y L. Davis. (Ed.). Trekking the shore. Changing coastlines and the antiquity of coastal settlement (pp.305-331). California, USA: Springer.

Okumura, M. M., Boyadjian, C. H. C., y Eggers, S. (2007). Auditory exostoses as an aquatic activity marker: a comparison of coastal and inland skeletal remains from tropical and subtropical regions of Brazil. American Journal of Physical Anthropology, 132, 558-567.

Oliva, G., González, L., Rial, P., Livraghi, E., Borrelli, P., y Oliva, G. (2001). Áreas ecológicas de Santa Cruz y Tierra del Fuego. Ganadería ovina sustentable en la Patagonia Austral.

Orquera, L. A., y Piana, E. (1999). La vida material y social de los Yámanas. Buenos Aires,Argentina: Eudeba

Orquera, L. A. (2005). Mid-Holocene littoral adaptation at the southern end of South America. Quaternary International, 132, 107-115.

Özbek, M. (2012). Auditory exostoses among the pre-pottery Neolithic inhabitants of Çayönü and Aşıklı, Anatolia; its relation to aquatic activities. International Journal of Paleopathology, 2, 181-186.

Pezo Lanfranco, L., Pezo Lanfranco, S., y Eggers, S. (2009). Exostosis auditiva como marcador osteológico de actividad acuática en poblaciones formativas de la costa norte del Perú. Revista Paleopatología, 6, 1-18.

Ponce, P., Ghidini, G., y González-José, R. (2008). External auditory exostosis "at the end of the world": the southernmost evidence according to the latitudinal hypothesis. Proceedings Eight Annual Conference of the British Association for Biological Anthropology and Osteoarchaeology. Brickel M, Smith M. (Ed.). Bar International Series 1743. Archaeopress, Oxford, 101-107.

Sakalinskas, V., y Jankauskas, R. (1993). Clinical otosclerosis and auditory exostoses in ancient Europeans (investigations of Lithuanian paleoosteological samples). Journal of Laryngology and Otology, 107, 489-491

Saletta, M. J. (2014). Excavando fuentes: la tecnología, 
subsistencia, movilidad y sistemas simbólicos en Shelk'nam, Yámana/Yaghan y Aonikenk entre los siglos XVI y XX analizados a partir de registros escritos y arqueológicos (Tesis Doctoral). Universidad de Buenos Aires, Argentina.

Santiago, F., Salemme, M., Suby, J., y Guichón, R. (2011). Restos humanos en el norte de Tierra del Fuego. Aspectos contextuales, dietarios y paleopatológicos. Intersecciones en Antropología, 12, 147-162.

Standen, V. G., Arriaza, B. T., y Santoro, C. (1997). External Auditory Exostosis in Prehistoric Chilean Populations: A Test of the Cold Water Hypothesis. American Journal of Physical Anthropology, 103, 119-129.

Tessone, A., Zangrando, A. F., Valencio, S., y Panarello, H. O. (2003). Análisis de isótopos en restos óseos humanos en la región del canal Beagle (Isla Grande de Tierra del Fuego). Revista Argentina de Antropología Biológica, 5(2), 33-43.

Timofeev, I., Notkina, N., y Smith, I. M. (2004). Exostoses of the external auditory canal: a long-term follow-up study of surgical treatment. Clinical of Otolaryngology, $29,588-594$.

Tivoli, A. (2014). Processing activities and differentiation of bird utilization during the Late Holocene in the Beagle Channel region (Southern South America). International Journal of Osteoarchaeology, 24(3), 397-406.

Tivoli, A., y Zangrando, AF. (2011). Subsistence variations and landscape use among maritime hunter-gatherers. A zooarchaeological analysis from the Beagle Channel (Tierra del Fuego, Argentina). Journal of Archaeological Science, 38(5), 1148-1156.

Trinkaus, E., y Wu, X. J. (2017). External auditory exostoses in the Xuchang and Xujiayao human remains: patterns and implications among eastern EurAsian middle and late Pleistocene crania. PLOS ONE, 12(12), 6-8. doi:https://doi.org/10.1371/journal.pone.0189390

Umeda, Y., Nakajima, M., y Yoshioka, H. (1989). Surfer's ear in Japan. Laryngoscope, 99, 639-641.

Velasco-Vazquez, J., Betancor-Rodriguez, A., Arnay de la Rosa, M., y Gonzalez-Reimers, E. (2000). Auricular exostoses in the prehistoric population of Gran Canaria. American Journal of Physical Anthropology, 112, 49-55.

Villotte, S., y Knüsel, C. J. (2015). External auditory exostoses and prehistoric aquatic resource procurement. Journal of Archaeological Science: Reports, 6, 633636. doi:https://doi.org/10.1016/j.jasrep.2015.05.013

Villotte, S., Stefanovic, S., y Knüsel, C. J. (2014). External auditory exostoses and aquatic activities during the Mesolithic and the Neolithic in Europe: results from a large prehistoric sample. Anthropologie, L/2, 1-17.

Wong, B. J., Cervantes, W., Doyle, K. J., Karamzadeh, A. M., Boys, P., Brauel, G., y Mushtaq, E. (1999). Prevalence of external auditory canal exostoses in surfers. Archeaology and Otolaryngology, Head Neck Surgical, 125, 969 -972.

Yesner, D., Figuerero Torres, M., Guichón, R., y Borrero, L. (2003). Stable isotope analysis of human boneand ethnohistoric subsistence patterns in Tierra del Fuego. Journal of Anthropological Archaeology 22, 279-291.

Zangrando, F. (2009). Historia evolutiva y subsistencia de cazadores-recolectores maritimos de Tierra del Fuego (Tesis Doctoral). Universidad de Buenos Aires, Argentina

Zoltan, T. B., Taylor, K. S., y Achar, S. A. (2005). Health issues for surfers. American Family Physician, 71(12), 2313-2317. 